\title{
Genetic Improvement 2016 Chairs' Welcome
}

It is our great pleasure to welcome you to the 2nd Workshop on Genetic Improvement, associated with GECCO 2016. Our goal is to bring together research from across the globe to exchange ideas on using optimization techniques, particularly evolutionary computation such as genetic programming, to improve existing software. We invited short position papers to encourage the discussion of new ideas and recent work in addition to longer and more concrete submissions. The call for participation invited GI work on automatic bug fixing; improving functionality; improving non-functional properties such as efficiency, memory and energy consumption; "plastic surgery" by transplanting functionality from other existing code to host software; and automatically specializing generic software for dedicated tasks. As you will see, we have accepted papers in most of these areas as well as papers on compiler optimization and position papers on new GI applications, namely, in the areas of formal software verification and code obfuscation.

We had submissions from Asia, Europe, Australia and North America. Our reviewers helped us select 10 papers for the workshop.

Putting together GI-2016 was a team effort. We first thank the authors for providing the content of the program. We are grateful to the program committee who worked very hard in reviewing papers and providing feedback for authors. Special thanks go to William B. Langdon for helping us promote the event. We would also like to thank the GECCO 2016 workshop chairs for support in organizing the workshop. Finally, we thank our sponsors: GrammaTech, Westley Weimer and profits from the book "A Field Guide to Genetic Programming” by Riccardo Poli, William B. Langdon and Nicholas Freitag McPhee.

We hope that you will find this program interesting and thought provoking, and that the workshop will provide you with a valuable opportunity to share ideas with other researchers from institutions around the world. We hope that the keynote by Westley Weimer and our special session for students with leading researchers in the field will facilitate exciting new research directions. We expect that you will notice many areas of software engineering that are not covered. Our primary aim is to encourage you to participate. We hope for many questions along the lines of: "Can GI do X?" to which the reply may be: please try! Go one step better than the White Queen and do something considered to be impossible!
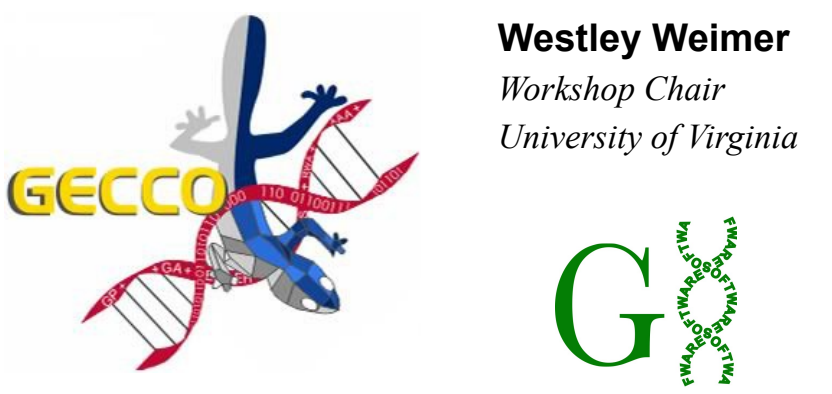

\author{
Justyna Petke \\ Workshop Chair \\ University College London
}

David R. White

Workshop Chair

University College London 


\section{Genetic Improvement 2016 Organization}

Justyna Petke (University College London, UK)

Chairs: Westley Weimer (University of Virginia, USA)

David R. White (University College London, UK)

Program Committee: Bobby R. Bruce (University College London, UK)

Nathan Burles (IBM, UK)

Brendan Cody-Kenny (Trinity College Dublin, Ireland)

Saemundur O. Haraldsson (University of Stirling, UK)

William B. Langdon (University College London, UK)

Claire Le Goues (Carnegie Mellon University, USA)

Justyna Petke (University College London, UK)

Eric Schulte (GrammaTech, USA)

Jerry Swan (University of York, UK)

Westley Weimer (University of Virginia, USA)

David White (University College London, UK)

John R. Woodward (University of Stirling, UK)

Sponsors: Westley Weimer (University of Virginia, USA)

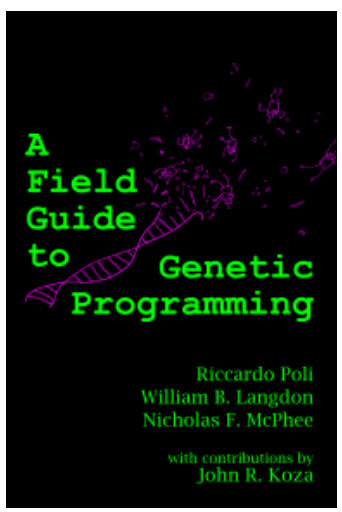

